\title{
Non-clinical factors associated with headache presentations in patients with psychological co-morbidities: a 7-year retrospective study
}

\author{
Chris O. Ifediora MBBS MPH FRACGP \\ Senior Lecturer, School of Medicine, Griffith University, Gold Coast Campus, QLD, Australia
}

\begin{abstract}
Background: Multiple factors potentially influence doctors’ decisions regarding neuroimaging for headaches in general practice and psycho-morbidities are one of such. Unfortunately, other non-clinical factors at play among psychological patients with headaches are poorly known and the need to identify these factors has become important in view of recent findings on the impact of psychological issues in managing headaches in primary care. The findings from this study are expected to help improve efficiency and confidence in managing headaches in this subset of patients.

Method: This is a 7-year retrospective study exploring headache presentations of patients aged $\geq 18$ in an Australian general practice setting from 1 January 2010 to 1 April 2017.

Results: A total of 517 patients were identified, with an average age of 45.5 +/- 16.2 years (range of 18 to 94 years). Psycho-morbidities were reported among 190 (36.8\%) patients. The female to male ratio was $72 \%$ to 28\%. Most cases of headaches were among those aged 40 to 59 years (199 or 38.5\%), while the least presentations were among the 18 to 24 year-olds (47 or $9.1 \%$ ). It was found that $40 \%$ fewer males had psycho-morbidities relative to females (OR 0.6 ; CI 0.39 to 0.91; p -0.02), while those aged between 40 and 59 years were more likely to have associated psycho-morbidities compared to other age groups (OR 1.68; CI 1.17 to 2.42; p -0.01).

Conclusion: General Practitioners should properly evaluate the psychosocial issues of all patients presenting with headaches for which neuroimaging is being considered, particularly middle-aged females. This is important given the recent report that neuroimaging for headache presentations among patients with associated psycho-morbidities are ordered more disproportionately and yet were less likely to have findings that can explain the headaches. A larger national or international study may be needed to further explore the identified associations.
\end{abstract}

\section{Keywords}

Decision-making, general practice, headache, mental health, person-centered healthcare, psychological, psychological comorbidities, primary care

\section{Correspondence address}

Dr. C.O. Ifediora, Senior Lecturer, School of Medicine, Griffith University, Gold Coast Campus, Parklands Drive, Southport, QLD 4215, Australia. E-mail: c.ifediora@griffith.edu.au

Accepted for publication: 8 May 2018

\section{Introduction}

Headaches are a common reason for primary care consultations in general practice [1-3] and account for up to $1.5 \%$ of all visits per year [1]. The social, economic and personal costs of headaches can be considerable [4,5].

Dangerous causes of headaches are, however, rare $[1,3,5]$ and most cases in general practice are treated only by physical examination, drug prescriptions [1] and reassurances [6]. However, where deemed relevant, General Practitioners (GPs) do refer patients for investigations such as neuroimaging (covering head and brain scans) or specialist opinions [6]. One of such cases that justify referrals is the presence of 'red flags', which include sudden, severe presentation of first-time headaches, or the recurrence of cases that are different to previous episodes [7]. They also include cases where headaches are exacerbated by coughing, sneezing, straining, eye movements and postural changes, as well as those associated with blurred vision, neck stiffness, rash, altered consciousness, confusion and focal neurological signs [7].

In the absence of these red flags, however, some patients are still referred for imaging scans and purported reasons for these might include 'pressure' from patients, uncertainties and the fear of medico-legal actions on the part of the doctors [5,8-13]. Multiple publications have established the existence of non-clinical factors that influence doctors' decisions to investigate or refer patients presenting with headaches [8-10,14-16] and ordering 
neuroimaging tests may simply be to help relieve patients' concerns [12,17]. One such concern may arise from patients with psychologically co-morbidities, with a recently completed paper reporting that patients with identified psycho-morbidities were $78 \%$ less likely to have findings that will explain their headaches following neuroimaging scans when compared to those without these co-morbidities $(p=0.03)$ [18]. The same study also reported that headache consultations involving patients with coexisting psychological conditions were likely to result in neuroimaging investigations, even though this particular finding narrowly missed statistical significance $(\mathrm{p}=0.08)$.

The need, therefore, arises for studies to investigate the characteristics of this subset of patients with psychological co-morbidities presenting with headaches, with the hope of identifying factors that will assist doctors in managing them. This study was undertaken to identify some of these factors and its findings are expected to provide GPs with some evidence-based frameworks to assist their decisionmaking on 'when' and 'who' to investigate, thereby boosting their confidence in managing headaches among psychological patients. Costs will also be saved for both governmental and private healthcare funders, as efficient decision-making guidelines developed with inputs from studies such as this will help minimize the combination of direct (through the avoidance of unnecessary imaging tests) and indirect (by saving time and other work-related implications) costs arising from neuroimaging for headaches. Finally, this study is expected to add to the existing database of studies on headaches in primary care, an area that has been surprisingly understudied and provide recommendations for both policy changes and future research into the management of headaches in general practice.

\section{Methods}

\section{Setting}

This is a 7-year retrospective study that explored all documented headache presentations in an Australian general practice setting between the $1^{\text {st }}$ of January 2010 and the $1^{\text {st }}$ of April 2017. It is part of a broader exercise that also explored the imaging patterns for headaches during the same time period. The focus was on a group of 3 geographically distinct, but administratively and functionally inter-connected general practice surgeries named 'Kennedy Drive Medical Centre (KDMC)', 'PKG Medical Centre' and the 'Bilambil Heights Medical Centre (BHMC)'. All 3 are located in the 'Tweed Shire Area' of Northern New South Wales (NSW), Australia, also called the 'Tweed Area' or simply 'The Tweed' [19].

'The Tweed' covers about 1,303 $\mathrm{km}^{2}$ and sits at the border of the states of NSW and Queensland, about 102 $\mathrm{km}$ to the South of Brisbane and $830 \mathrm{~km}$ North of Sydney [20]. Alone, the Tweed's population is approximately 90,000 [19]. However, the 3 surgeries are among a few others serving an estimated patient population of about $150,000[21,22]$ and patients that live in the Tweed, as well as the southern suburbs of the City of Gold Coast, all patronize them.

The records and files of all patients, as well as the management system of all the 3 surgeries, are inter-linked and, combined, they have a registered patient population of about 31,000, served by a maximum of 11 doctors at each particular time (7 in KDMC, 3 in PKG and 2 in BHMC).

\section{Demographics}

Even though the median age of the population in the Tweed Area was about 54 years according to the 2011 census, the larger 'Gold Coast-Tweed Area', which this study focused on, has a median age of 38 years, a number similar to the Australian average of 37 years [20]. The older median age in the Tweed is because the area is a hub of retirees, hosting about 14 separate retirement villages [20]. About $87 \%$ of the adult population in the Gold CoastTweed Area is employed (either full or part-time), with a male to female ratio of 51:49 and the child to family ratio of $2: 1$ [20]. Over $80 \%$ of the residents are Caucasians, while $87 \%$ have English as their dominant first language [20].

\section{Inclusion and Exclusion criteria}

The Inclusion Criteria included all adults $\geq 18$ years of age at the time of their presentation, who saw a doctor within the defined period in any of the 3 identified practices, with headache as a major complaint (whether imaging was ordered or not). Headaches of all kinds were included, provided it was the main reason for the consultation. All potential forms of neuroimaging were allowed, including Computed Tomography (CT), Magnetic Resonance Imaging (MRI), Magnetic Resonance Angiography (MRA), Ultrasound (USS) and X-rays.

The patient's age as at the time of the data collection, along with any existing psychological co-morbidity (psycho-morbidity), was also recorded. Psycho-morbidities include depression, anxiety, post-traumatic stress disorder (PTSD), bipolar diseases, stress and others. To be included, these morbidities must already have been diagnosed and documented as at the time of the consultation for the headache and there must be evidence of treatment, which can either be pharmacological (with medications) or psychological (backed by evidence of correspondences with psychologists and psychiatrists). These criteria were included to avoid spurious or transient forms of psychological disorders, for instance, cases used as excuses for work absence certificates, minor grief situations, transient work stress et cetera.

A number of the patients had more than one headache presentations as well as multiple head scans over the 7year period. In these cases, one documented presentation of headache is enough to allow inclusion and a single order of head imaging, provided that the order was directly related to the headache, were included in the analysis. 
Table 1 Demographics and characteristics of patients that presented with headaches over a 7-year period in an Australian general practice setting $(n=517)$

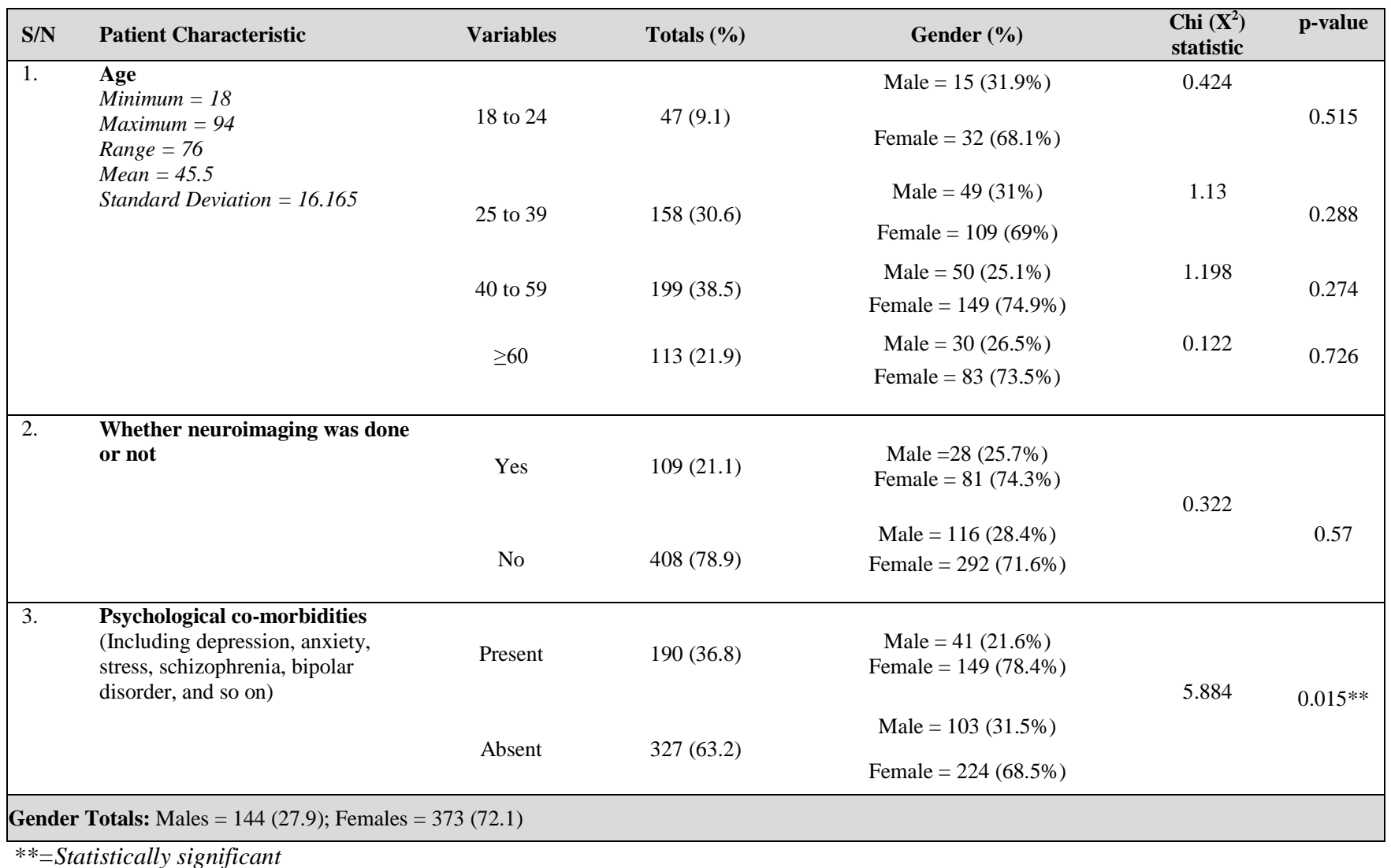

Table 2 Binary Logistics Regression of associations between the presence of psychological comorbidities (Outcome or Dependent Variable) and some characteristics of the patients that presented with headaches in an Australian general practice setting over a 7-year period $(n=517)$

\begin{tabular}{|c|c|c|c|c|c|c|}
\hline \multirow[b]{2}{*}{$\mathrm{S} / \mathbf{N}$} & \multirow[b]{2}{*}{ Patient Characteristics } & \multirow{2}{*}{$\begin{array}{c}\text { Variables } \\
\text { (Predictor/(Independent) }\end{array}$} & \multirow{2}{*}{$\begin{array}{l}\text { Odds ratio } \\
\text { (OR) }\end{array}$} & \multicolumn{2}{|c|}{ 95\% CI } & \multirow{2}{*}{$\begin{array}{l}\text { Significance } \\
\text { (p-value) }\end{array}$} \\
\hline & & & & Lower & Upper & \\
\hline 1 & Gender & Male Vs Female & 0.598 & 0.394 & 0.908 & $0.016^{* *}$ \\
\hline 2 & Age Group 1 & “ $\geq 18-24 ”$ Vs. others & 1.58 & 0.812 & 3.075 & 0.178 \\
\hline 3 & Age Group 2 & “25-39” Vs. Others & 0.818 & 0.552 & 1.212 & 0.316 \\
\hline 4 & Age Group 3 & “40-59” Vs. Others & 1.679 & 1.165 & 2.42 & $0.005 * *$ \\
\hline 5 & Age Group 4 & $" \geq 60 "$ Vs. Others & 0.759 & 0.487 & 1.183 & 0.223 \\
\hline
\end{tabular}

**=Statistically significant; $\quad *=$ Narrowly missed statistical significance

\section{Data Collection and Patient selection}

Data collection was performed between May and June of 2017. The "search tool" in the Medical Director ${ }^{\circledR}$ (MD) software was used to identify all the patients seen in the defined period who met the study criteria. The search filter was broadened to include all forms of headaches and migraines with all their identified variations, including Cervicogenic, Chronic, Cluster, Tension, Migraine (Common, Classical, Cluster, Migrainous Aura), Post Head Injury, among others. All patients identified were 
then manually reviewed to ensure that they met all of the inclusion-exclusion criteria.

\section{Data analysis}

Analysis was with the IBM SPSS Version 24, with the aim of answering the key research questions. Both the Chisquared $4 \times 4$ Analysis and the Binary Logistics Regression (BLR) were used as necessary. The probability (p-value) was set at $<0.05$, with a $95 \%$ Confidence Interval reported where deemed fit. One Outcome (Dependent) Variable, which was the 'Presence or Absence Of PsychoMorbidities', was tested against 5 Predictor (Independent) Variables, including 'Gender' and the Age Groups of ' $\geq 18$ 24 ', '25-39', '40-59' and ' $\geq 60$ '.

\section{Ethical Considerations}

Ethical Approval was obtained from the Human Research Ethics Committee (HREC) of the Griffith University (GU Ref No: 2017/354) prior to the commencement of our study.

\section{Results}

A total of 517 patients met the inclusion criteria and were part of the study. The relevant basic characteristics of these patients are shown in Table 1 and reveals that the average age was 45.5 years (SD 16.165), with a range of 18 to 94 years. Females outnumbered the males (72\% to $28 \%)$ and most (199 or 38.5\%) of the patients were aged 40 to 59, while the least headache presentations were among those aged 18 to 24 years (47 or 9.1\%). Head imaging was ordered for 109 or $21.1 \%$ (91 CT scans and 18 MRIs) patients, while 190 (36.8\%) had psychological comorbidities.

Table 1 demonstrates that there were no significant gender differences across all age brackets or with the ordering of neuroimaging. However, significantly more females with headaches had associated psychological comorbidities compared to males $\left(\mathrm{X}^{2}[1, \mathrm{~N}=517]=5.88\right.$, $\mathrm{p}=0.02)$.

Table 2 presents the findings of the BLR analysis that explored the associations between the patients with psychological co-morbidities and a number of patient characteristics. In line with the gender association already identified in Table 1 , male patients presenting with headaches were $40 \%$ less likely to have psychological issues when compared to females (OR 0.6; CI 0.39 to 0.91; $\mathrm{p}-0.02)$. However, it was further found that patients aged 40-59 years who presented with headaches were just over one-and-half times more likely to have co-existing psychological problems compared to other age groups (OR 1.68; CI 1.17 to 2.42; p -0.01).

\section{Disc ussion}

The observation that a large majority (78.9\%) of the entire 517 patients seen for headaches (Table 1) did not require imaging appears to be consistent with reports of multiple studies, which all found that drug prescriptions [1], physical examinations and history $[1,23]$ are the common methods of managing headaches in primary care and not head imaging.

It is not entirely surprising that less males with headaches have associated psycho-morbidities compared to males $(p=0.02)$, given that that more female patients in Australia present with headaches [13] and have more mental health problems when compared to males [24]. Also, even though the age group of 25-44 years required head scans the most in Australia as reported in publications by the Bettering the Evaluation and Care of Health (BEACH) study [13] and that of a recent paper [18], our work found that, among the subset of patients with psychological co-morbidities presenting with headaches, the older age group of 40-59 years predominate (p -0.01). Our paper appears to be the first to identify this relationship and this, along with the finding on gender, might have important implications for policy and practice as discussed below.

The above two associations (gender and age) might help alert doctors to the psychological conditions of females aged 40 to 59 years when they present with headaches in general practice. While it is important to do this for all patients, this study has focussed its attention on this age and gender demographic. Such attention is even more important given the findings of a recent paper [18], which concluded that neuroimaging for headaches in which psycho-morbidities is significantly associated with a less chance of findings that can explain headaches $(\mathrm{p}=0.03$ ) and that such consultations may result in the ordering of more neuroimaging investigations $(\mathrm{p}=0.08)$.

Caution is advised in the application of these findings though, given the potential limitations of this work (see below). A larger study may help evaluate these observations, but, until that is conducted, the merits from the findings of this work should not be ignored given the significant policy and practice implications.

One limitation of this study is the limited scope of this work, given that only three practices (functionally and geographically equivalent to one) were involved. Even though the practice is relatively large, the findings may not represent the pattern Australia-wide and a larger, more national study is necessary. An additional limitation is that some patients with psycho-morbidities might not have been diagnosed accordingly, while others might not have the diagnosis properly documented in the clincal records. Since this study relied on such records, such cases might not have been identified. However, the 7-year period over which this study was conducted is a duration sufficient to ensure that most patients with clinically significant psychomorbidities would have been picked up at some point and documented accordingly. This factor therefore minimizes the potential effect of this limitation on the overall study outcome. A final potential limitation is the possibility of human error in data collection. To control for this, all 
efforts were made to check the collated data twice in other to minimize any such potential impact.

\section{Conclusions and Recommendations}

This study concludes that just over 1-in-3 (36.8\%) of patients presenting with headaches over a 7-year period in an Australian general practice setting had associated psycho-morbidities and that males were $40 \%$ less likely to have associated psychological issues when compared to females (OR 0.6; p -0.02). In addition, patients aged 40-59 years who presented with headaches were just over one and half times more likely to have co-existing psychological problems compared to other age groups (OR 1.68; p -0.01).

It is recommended that medical practitioners should properly evaluate the psychosocial issues of all patients presenting with headaches for which neuroimaging is being considered, particularly middle aged females. The need for this is even more important given that a related paper had observed a statistically significant observation, namely that headache presentations among patients with associated psycho-morbidities that have undergone neuroimaging were less likely to have findings reported in their brain scans which can explain their headaches

\section{Acknowledgements and Conflicts of Interest}

I acknowledge the immense support from Mrs. Maureen Firehock, the Managing Director and CEO of all three practices used in this work (Kennedy Drive, PKG and Bilambil Heights Medical Centers, Tweed Heads, Australia). I am also appreciative of the efforts of all the staff in these surgeries who assisted with the data collection and other logistics needed to make this study a success. There was no external source of funding for this study. I declare no conflicts of interest.

\section{References}

[1] Frese, T., Druckrey, H. \& Sandholzer, H. (2014). Headache in General Practice: Frequency, Management, and Results of Encounter. International Scholarly Research Notices 2014:169428.

[2] Rasmussen, B.K., Jensen, R., Schroll, M. \& Olesen, J. (1991). Epidemiology of headache in a general population - a prevalence study. Journal of Clinical Epidemiology 44 (11) 1147-1157.

[3] Latinovic, R., Gulliford, M. \& Ridsdale, L. (2006). Headache and migraine in primary care: consultation, prescription, and referral rates in a large population. Journal of Neurology, Neurosurgery \& Psychiatry 77 (3) 385-387.

[4] Wonderling, D., Vickers, A.J., Grieve, R. \& McCarney, R. (2004). Cost effectiveness analysis of a randomised trial of acupuncture for chronic headache in primary care. British Medical Journal 328 (7442) 747.

[5] Kernick, D., Stapley, S. \& Hamilton, W. (2008). GPs' classification of headache: is primary headache underdiagnosed? British Journal of General Practice 58 (547) 102-104.

[6] O'Flynn, N. \& Ridsdale, L. (2002). Headache in primary care: how important is diagnosis to management? British Journal of General Practice 52 (480) 569-573.

[7] Beran, R.G. (2014). Management of chronic headache. Australian Family Physician 43 (3) 106.

[8] Stanley, I.M., Peters, S. \& Salmon, P. (2002). A primary care perspective on prevailing assumptions about persistent medically unexplained physical symptoms. The International Journal of Psychiatry in Medicine 32 (2) 125-140.

[9] Page, L., Howard, L., Husain, K., Tong, J., Dowson, A.J., Weinman, J. \& Wessely, Q.C. (2004). Psychiatric morbidity and cognitive representations of illness in chronic daily headache. Journal of Psychosomatic Research 57 (6) 549-555.

[10] Couchman, G.R., Forjuoh, S.N., Rajab, M.H., Phillips, C.D. \& Yu, J. (2004). Nonclinical factors associated with primary care physicians' ordering patterns of magnetic resonance imaging/computed tomography for headache 1. Academic Radiology 11 (7) 735-740.

[11] Kernick, D.P., Ahmed, F., Bahra, A., Dowson, A., Elrington, G., Fontebasso, M., Giffin, N.J., Lipscombe, S., MacGregor, A., Peatfield, R., Weatherby, S. \& Whitmarsh, T. (2008). Imaging patients with suspected brain tumour: guidance for primary care. British Journal of General Practice 58 (557) 880-885.

[12] Frishberg, B.M., Rosenberg, J.H., Matchar, D.B. et al. (2000). Evidence-based guidelines in the primary care setting: neuroimaging in patients with nonacute headache. St Paul, MN: US Headache Consortium.

[13] Britt, H., Ng, A. \& Charles, J. (2005). Presentations of headache in Australian general practice. Australian Family Physician 34 (8) 618-619.

[14] American Academy of Neurology. (2008). Report of the Quality Standards Sub-Committee of the American Academy of Neurology. The utility of neuro imaging in the evaluation of headache in patients with normal neurological examinations.

[15] Morgan, M., Jenkins, L. \& Ridsdale, L. (2007). Patient pressure for referral for headache: a qualitative study of GPs' referral behaviour. British Journal of General Practice 57 (534) 29-35.

[16] Ridsdale, L., Clark, L.V., Dowson, A.J., Goldstein, L.H., Jenkins, L., McCrone, P., Morgan, M. \& Seed, P.T. (2007). How do patients referred to neurologists for headache differ from those managed in primary care? British Journal of General Practice 57 (538) 388-395.

[17] De Luca, G. \& Bartleson, J. (2010). When and how to investigate the patient with headache. Paper presented at: Seminars in neurology 2010.

[18] Ifediora, C.O. (2018). Insights into radiographic investigations for headaches in general practice. Family Practice 35 (4) 512-519. 
[19] Tweed Shire Council. The Tweed. (2017).; Available at: http://www.tweed.nsw.gov.au/TheTweed. Accessed July 17th, 2017.

[20] Australian Bureau of Statistics. (2017). 2011 Census QuickStats: Tweed Heads. Available at: http://www.censusdata.abs.gov.au/census_services/getprod uct/census/2011/quickstat/SSC12332. Accessed September 26th, 2017.

[21] Citipedia. Gold Coast, Australia - list of cities, towns, villages. (2017). Available at:

http://www.citipedia.info/province/general/Australia_Gold +Coast. Accessed July 17th 2017.

[22] City of Gold Coast. Gold Coast population fact sheets. (2017). Available at:

http://www.goldcoast.qld.gov.au/social-planning-anddevelopment-factsheets-2006-2010-5152.html. Accessed July 17th, 2017.

[23] Becker, L., Iverson, D.C., Read, F., Calogne, N., Miller, R. \& Freeman, W. (1987). A study of headache in North American primary care: Report for the Ambulatory Sentinel Practice Network. Wollongong, Australia: University of Wollongon research Online.

[24] Department of Health. (2017). Prevalence of mental disorders in the Australian population. Available at: http://www.health.gov.au/internet/publications/publishing. nsf/Content/mental-pubs-m-mhaust2-toc mental-pubs-mmhaust2-hig mental-pubs-m-mhaust2-hig-pre. Accessed 4th November, 2017. 